\title{
AF-MSCs fate can be regulated by culture conditions
}

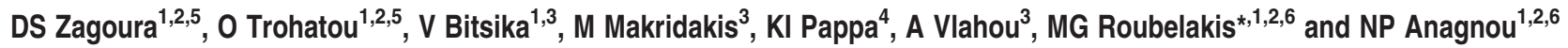

Human mesenchymal stem cells (hMSCs) represent a population of multipotent adherent cells able to differentiate into many lineages. In our previous studies, we isolated and expanded fetal MSCs from second-trimester amniotic fluid (AF) and characterized them based on their phenotype, pluripotency and proteomic profile. In the present study, we investigated the plasticity of these cells based on their differentiation, dedifferentiation and transdifferentiation potential in vitro. To this end, adipocyte-like cells (AL cells) derived from AF-MSCs can regain, under certain culture conditions, a more primitive phenotype through the process of dedifferentiation. Dedifferentiated AL cells derived from AF-MSCs (DAF-MSCs), gradually lost the expression of adipogenic markers and obtained similar morphology and differentiation potential to AF-MSCs, together with regaining the pluripotency marker expression. Moreover, a comparative proteomic analysis of AF-MSCs, AL cells and DAF-MSCs revealed 31 differentially expressed proteins among the three cell populations. Proteins, such as vimentin, galectin-1 and prohibitin that have a significant role in stem cell regulatory mechanisms, were expressed in higher levels in AF-MSCs and DAF-MSCs compared with AL cells. We next investigated whether AL cells could transdifferentiate into hepatocyte-like cells (HL cells) directly or through a dedifferentiation step. AL cells were cultured in hepatogenic medium and 4 days later they obtained a phenotype similar to AF-MSCs, and were termed as transdifferentiated AF-MSCs (TRAF-MSCs). This finding, together with the increase in pluripotency marker expression, indicated the adaption of a more primitive phenotype before transdifferentiation. Additionally, we observed that AF-, DAF- and TRAF-MSCs displayed similar clonogenic potential, secretome and proteome profile. Considering the easy access to this fetal cell source, the plasticity of AF-MSCs and their potential to dedifferentiate and transdifferentiate, AF may provide a valuable tool for cell therapy and tissue engineering applications.

Cell Death and Disease (2013) 4, e571; doi:10.1038/cddis.2013.93; published online 4 April 2013

Subject Category: Experimental Medicine

Human mesenchymal stem cells (hMSCs) have been isolated from adult tissues, such as bone marrow (BM) $)^{1,2}$ and adipose tissue ${ }^{3}$ as well as from fetal sources, including amniotic fluid (AF) ${ }^{4-7}$ Wharton's jelly ${ }^{8}$ and umbilical cord blood. ${ }^{9,10}$ MSCs are multipotent cells capable of generating osteogenic, adipogenic and chondrogenic cells in response to specific culture conditions in vitro. ${ }^{1,4,6,7,11,12}$

Recently, our group has identified and studied MSCs from human second-trimester AF, obtained during routine amniocenteses for prenatal diagnosis. ${ }^{6,7}$ The AF-MSC population is gaining attention with regard of belonging to an intermediate developmental stage between embryonic and adult stem cells. ${ }^{4-7,13}$ Interestingly, AF-MSCs appear to express pluripotency markers such as Sox-2, Oct-4 and Nanog. ${ }^{6,7,13} \mathrm{We}$ documented that these cells exhibited high proliferation rate in culture, were karyotypically stable when cultured ex vivo and differentiated in vitro not only into cell types derived from mesoderm but also into endoderm-derived cells, such as hepatocytes. ${ }^{6,7,12}$ This multipotential differentiation capability of AF-MSCs can be utilized for giving rise to a variety of differentiated cell types for tissue repair and regeneration. ${ }^{4,12}$ To this end, we have recently shown the therapeutic effect of AF-MSCs and hepatic progenitors, derived from AF-MSCs, in $\mathrm{CCl}_{4}$ acute hepatic failure mouse model, and have investigated the mechanism of their action at the site of injury ${ }^{12}$ without generating teratomas in vivo. ${ }^{4,5,12}$

During differentiation process, culture conditions, including specific growth factors, cytokines and extracellular matrix components, may have an important role in the determination of the stem cell fate by switching from the self-renewal to a differentiation stage and vice versa. ${ }^{11,14,15}$ This may happen through various processes including dedifferentiation or transdifferentiation. ${ }^{11}$ During dedifferentiation a terminally differentiated cell returns to a more primitive state, whereas the term transdifferentiation describes the process where a fully differentiated cell acquires characteristics of other cell types by switching its phenotype. ${ }^{11,16}$ Several studies have documented that during dedifferentiation a downregulation of

\footnotetext{
${ }^{1}$ Laboratory of Biology, University of Athens School of Medicine, Athens, Greece; ${ }^{2}$ Cell and Gene Therapy Laboratory, Centre of Basic Research II, Biomedical Research Foundation of the Academy of Athens (BRFAA), Athens, Greece; ${ }^{3}$ Biotechnology Laboratory, Centre of Basic Research, Biomedical Research Foundation of the Academy of Athens (BRFAA), Athens, Greece and ${ }^{4}$ First Department of Obstetrics and Gynecology, University of Athens School of Medicine, Athens, Greece ${ }^{*}$ Corresponding author: MG Roubelakis, Laboratory of Biology, Medical School, University of Athens, Athens 11527 , Athens 11527, Greece Tel: + 302107462 145, + 302106597 013; Fax: +30 2106597 545; E-mail: roubel@ med.uoa.gr or mroubelaki@bioacademy.gr

5oint first authors.

${ }^{6}$ Joint Senior Authors.

Keywords: AF-MSCs; differentiation; dedifferentiation; transdifferentiation; culture conditions

Abbreviations: hMSCs, human mesenchymal stem cells; AF, Amniotic fluid; AF-MSCs, mesenchymal stem cells derived from amniotic fluid; DAF-MSCs, dedifferentiated AF-MSCs; TRAF-MSCs, transdifferentiated AF-MSCs; AL cells, adipocyte-like cells; OL cells, osteocyte-like cells; HL cells, hepatocyte-like cells; SSEA-4, stage-specific embryonic antigen 4; Sox-2, SRY-sex determination SRY region Y-box2; Oct-4, octamer-binding transcription factor 4; Nanog, homeobox transcription factor Nanog

Received 02.10.12; revised 04.2.13; accepted 20.2.13; Edited by Y Shi
} 
lineage-specific genes and an upregulation of stemness genes occur, which is evident for the regaining of stem cell phenotype. ${ }^{14,17,18}$ On the other hand, it has been demonstrated that transdifferentiation capability of hMSCs is related either to cell heterogeneity or cell fusion. ${ }^{11}$ More importantly, cell-based therapy has been focused on the investigation of the processes of dedifferentiation and transdifferentiation as potential therapeutic strategies. ${ }^{16}$

The fetal origin and the unique characteristics of AF-MSCs make them an advantageous mesenchymal stem cell population for studying the cellular and molecular mechanisms that are activated during the process of differentiation. In the present study, we have developed an in vitro differentiation system to analyze the cellular and molecular events involved during the processes of differentiation, dedifferentiation and transdifferentiation of AF-MSCs. Herein, we attempt to answer two fundamental questions: (a) whether in vitro differentiation is reversible and (b) whether committed progenitors derived from AF-MSCs can switch their phenotype to another cell type directly or through a more primitive phenotype.

\section{Results}

AL cells, derived from AF-MSCs, are able to dedifferentiate into a more primitive cell type. In the present study, cell pellets from six second-trimester human AF samples were plated according to previous protocols. ${ }^{6,7}$ Plastic-adherent colonies of AF-MSCs began to appear 8-12 days after the initial plating. Then, spindle-shaped (SS) AF-MSCs were isolated and further expanded up to 40-50 passages with normal karyotype and high proliferation capacity. ${ }^{6,7,12,19}$ AF-MSCs were tested for MSC marker expression and were positive for CD73, CD90, CD105 and CD166 antigens as well HLA-ABC, but not for the hematopoietic marker CD45 and the HLA-DR antigen. ${ }^{6,7,12,19}$

Previously, it has been reported that AF-MSCs were able to differentiate into adipocytes, osteocytes, chondrocytes, hepatocytes and neurocytes, representing in this way cell types originating from all three germ layers. ${ }^{6,7,12}$ Indeed, after 2 weeks in culture in adipogenic medium, AF-MSCs exhibited some typical characteristics of cultured adipocytes, and thus were termed as adipocyte-like cells (AL cells). ${ }^{6}$

Initially, we assessed whether $\mathrm{AL}$ cells derived from AF-MSCs could dedifferentiate to a more primitive stem celllike stage. As previously reported, AF-MSCs express embryonic-related stem cell markers, such as stage-specific embryonic antigen 4 (SSEA-4), SRY-sex determination SRY region Y-box2 (Sox-2), octamer-binding transcription factor 4 (Oct-4) and homeobox transcription factor Nanog. ${ }^{6,7}$ It was observed that during adipogenic induction of AF-MSCs, SSEA-4, Sox-2, Oct-4 and Nanog expression levels were gradually decreased after 2 weeks in adipogenic medium (Figure 1a i). For this reason, the expression of the embryonic stem cell-related markers were examined at different time points during the dedifferentiation process of AL cells into an uncommitted cell type. For dedifferentiation studies, AL cells were divided into three groups. One group was cultured in AF-MSC medium for 1 week, whereas the second and the third group were placed in culture in AF-MSC medium for 2 and 3 weeks, respectively.
Interestingly, $A L$ cells cultured in AF-MSC medium for 2 weeks exhibited similar expression levels of SSEA-4 (percentage of SSEA-4 expression, $72.49 \pm 8.41$ ) to AF-MSCs (percentage of SSEA-4 expression, $82.69 \pm 13.89$; Figure 1a ii). In addition, the pluripotency markers Sox-2 $(P=0.938$, Student's $t$-test $)$, Oct-4 $(P=0.064$, Student's $t$-test $)$ and Nanog ( $P=0.184$, Student's $t$-test) were expressed in similar levels in AF-MSCs and AL cells that have been cultured in AF-MSC medium for 2 weeks (Figure 1a ii). At this stage, cells were termed as dedifferentiated AF-MSCs (DAF-MSCs). DAF-MSCs showed higher expression levels for Oct-4, Sox-2 and Nanog compared with AL cells, documenting a more primitive phenotype of these cells (Figure 1a). Their phenotype was altered to fibroblast-like and lost their ability to form small lipid droplets, positive for Oil Red O staining (Figure 1b). Additionally, DAF-MSCs exhibited lower levels of expression for adipogenic markers, such as peroxisome proliferator-activated receptor $\gamma \quad(\operatorname{PPAR} \gamma)$ and lipoprotein lipase (LPL) compared with AL cells (Figure 1c).

Comparison of the mitochondrial mass and lysosomal activity in AF-MSCs, AL cells and DAF-MSCs was performed by staining the cells with either MitoTracker or LysoTracker fluorescent dyes (Molecular Probes Inc., Eugene, OR, USA; Figure 2a). MSCs exhibited decreased mitochondrial tracker dye/lysosome tracker dye ratio upon transitioning from the stem cell to the differentiated state, implying a possible alteration in the lysosomal activity. ${ }^{20,21}$ Thus, the ratio of mitochondrial to lysosomal fluorescence was higher in AF-MSCs (1.316 \pm 0.24$)$ and DAF-MSCs (1.58 \pm 0.51$)$ compared with $A L$ cells $(0.602 \pm 0.071)$ (Figure $2 b)$. In addition, DAF-MSCs were still able to further differentiate under appropriate culture conditions into AL, osteocyte-like (OL) and hepatocyte-like cells (HL) (Supplementary Figure 1a).

Differentially expressed proteins in AF-MSCs, DAFMSCs and AL cells. A comparison of the proteome profile of AF-MSCs, AL cells and DAF-MSCs was conducted, following their separation by $2 \mathrm{DE}$ on $\mathrm{pH}$ range of 4-7 linear strips and mass spectrometry (MS; Figure 3a). At least three gels per category were included in the analysis and representative gel images are shown in Figure 3a. Thirty one proteins were identified as differentially expressed (over 1.5-fold and $P<0.05$, Student's t-test; Supplementary Table 2). More particularly, a number of proteins was identified in AF-MSCs only, including 14-3-3 protein $\beta / \alpha$, nucleoside diphosphate kinase $\mathrm{A}$ (NDKA) and tubulin- $\beta$ chain 5 (TUBB), whereas collagen $\alpha-1(\mathrm{I})$ chain (COL1A1) and dihydrolipoyllysine-residue succinyltransferase (DLST) were present in DAF-MSCs and $\mathrm{AL}$ cells only, respectively (Supplementary Table 3). On the other hand, specific proteins were downregulated in AF-MSCs and DAF-MSCs in comparison with AL cells, such as galectin-1 (LEG-1) (Figure $3 \mathrm{~b} \mathrm{i}$ ), which has an important role in cell differentiation $^{22}$ and prohibitin (PHB) (Figure $3 b$ ii), a key molecule in mitochondrial function, cell proliferation and development. ${ }^{23}$ However, eukaryotic translation initiation factor-3 (EIF3I) ${ }^{24}$ (Figure 3b iii) (Supplementary Table 2) was upregulated in AF-MSCs and DAF-MSCs compared with AL cells. More interestingly, protein, such as vimentin (VIME; Figure 3b iv), which has a specific role in cell attachment, migration and 
a
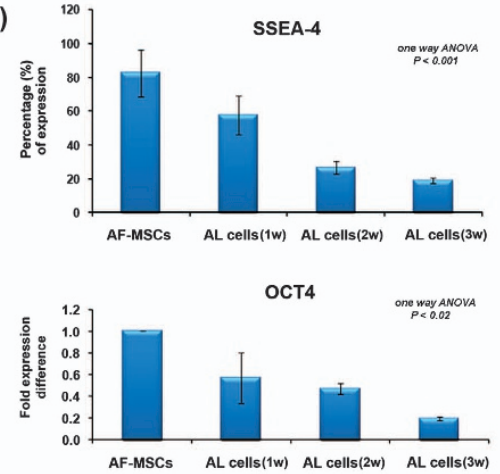

(ii)
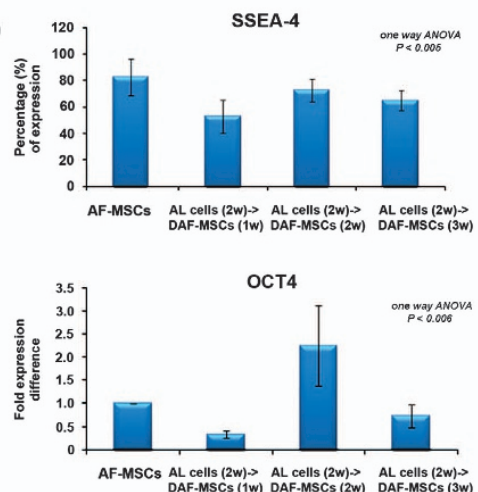

AF-MSCs

(i)

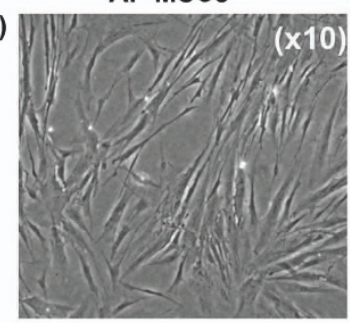

(ii)
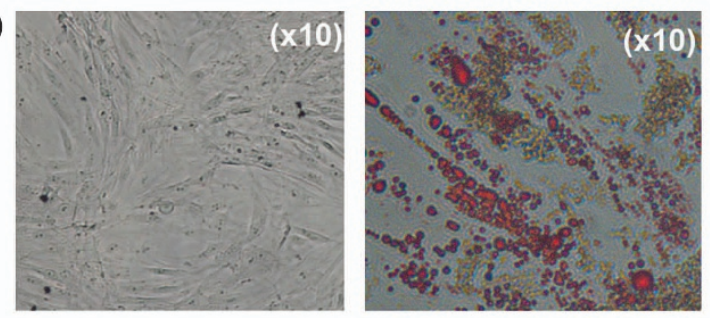

sox2
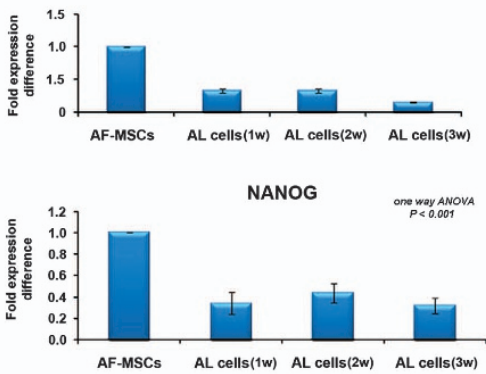

$\operatorname{sox} 2$
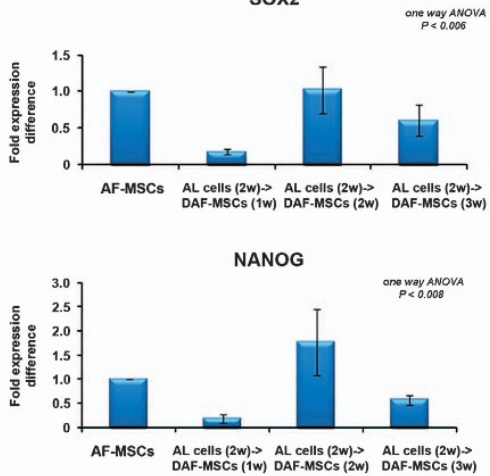

c

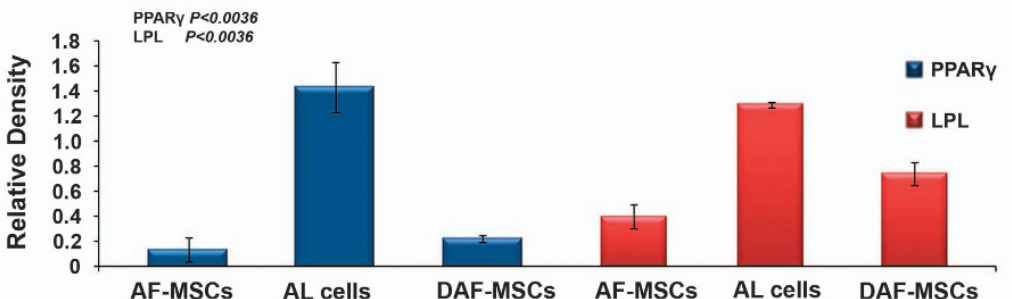

Figure 1 Dedifferentiation potential of AL cells. (a) (i) SSEA-4 expression levels were examined by FACS analysis, and Sox-2, Oct-4 and Nanog expression levels by Real-time PCR analysis genes during adipogenic induction of AF-MSCs. (ii) AF-MSCs and DAF-MSCs induced at different time points were analyzed for the expression of SSEA-4 by FACS analysis and for the expression of Sox-2, Oct-4 and Nanog by real-time PCR analysis. Data are presented as the mean \pm S.D. of three independent experiments, and were statistically analyzed by one-way ANOVA. (b) (i) Morphology of AF-MSCs, AL cells and DAF-MSCs in culture, and (b) (ii) after Oil Red O staining. (c) Semiquantitative PCR analysis for PPAR $\gamma$ and LPL on AF-MSCs, AL cells and DAF-MSCs. Data are presented as the mean \pm S.D. of three independent experiments and were statistically analyzed by one-way ANOVA 

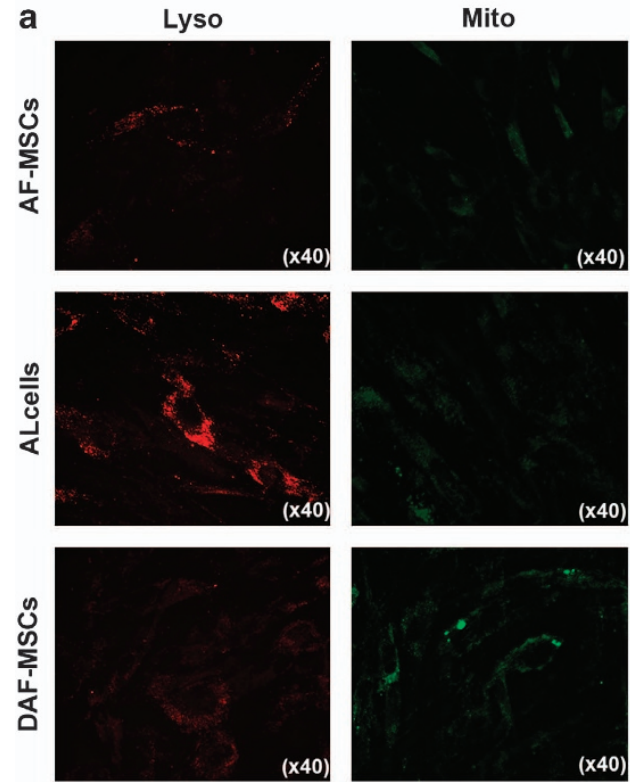

b

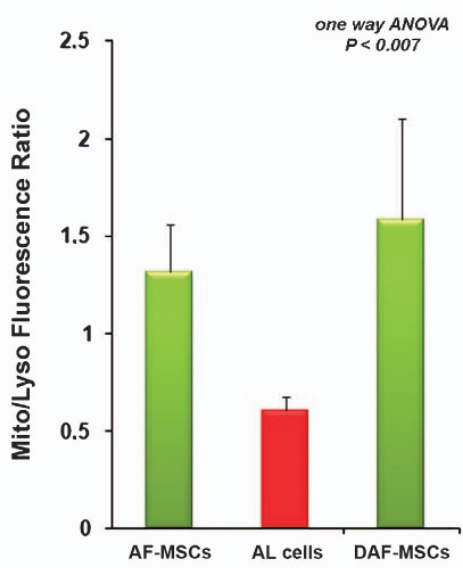

Figure 2 Metabolic activity and expression pattern of AF-MSCs, AL cells and DAF-MSCs. (a) Labeled lysosomes (red) and mitochondria (green) in AF-MSCs, AL cells and DAF-MSCs. (b) MitoTracker/LysoTracker ratios for the three cell types. Data are presented as the mean \pm S.D. of 10 images per cell category and were statistical analyzed by one-way ANOVA

signaling, ${ }^{25}$ was identified in AF-MSCs and DAF-MSCs only. Similarly, proteins with a regulatory role in protein synthesis such as, ATP synthase (ATPB; Figure 3b v), reticulocalbin-3 $(\mathrm{RCN} 3)^{26}$ (Figure $\left.3 \mathrm{~b} \mathrm{vi}\right)$, protein disulfide-isomerase $(P D I A 1)^{27}$ (Figure $3 b$ vii) and the metabolic protein glutathione S-transferase P1 (GSTP1; Figure 3b viii) were also identified in AF-MSCs and DAF-MSCs, suggesting that these two cell types were exhibiting a similar proteomic profile.

In order to verify the two-dimensional gel electrophoresis (2DE) results, western blotting analysis was performed for GSTP1 and PHB proteins. Bands of 32 and $26 \mathrm{kDa}$, corresponding to GSTP1 and PHB proteins, respectively, were detected in similar expression levels in AF-MSCs and DAF-MSCs (Figure 3c).

AL cells, derived from AF-MSCs, were capable to transdifferentiate into HL cells through a more primitive cell type. We further examined whether transdifferentiation of $\mathrm{AL}$ cells into $\mathrm{HL}$ cells happens directly or through the process of dedifferentiation, into a more primitive phenotype (Figure 4a). For this reason, AL cells were induced to hepatogenesis for 21 days. The transdifferentiation process was evaluated by Oct-4 expression analysis at different time points. An increase in the expression of Oct- 4 was detected after the 4th day in hepatogenic medium (fold expression difference: $0.19 \pm 0.04$ ) compared with AL cells (fold expression difference: $0.07 \pm 0.01$ ), suggesting that cells before differentiating into $\mathrm{HL}$ cells, may return to an uncommitted developmental stage (Supplementary Figure 2). Cells at this stage (4th day) exhibited a spindle-shaped morphology and were termed as transdifferentiated AF-MSCs (TRAF-MSCs; Figure $4 \mathrm{~b}$ i). Functional analysis revealed that only a small percentage of TRAF-MSCs $(11.1 \% \pm 6.2)$ were positive for Oil Red $\mathrm{O}$ staining versus $\mathrm{AL}$ cells $(43.2 \% \pm 12.11$;
Figure 4b I, ii). Additionally, only few TRAF-MSCs were able to store glycogen $(6.75 \% \pm 2.87)$ and uptake low-density lipoprotein (LDL; $2.2 \pm 0.75$, fluorescence intensity), compared with $\mathrm{HL}$ cells $(32 \% \pm 6.48$ for glycogen storage and $15.6 \pm 3.3$ fluorescence intensity for LDL uptake; Figure 4b iii, iv). Notably, TRAF-MSCs were still able to further differentiate, under appropriate culture conditions into $A L$ and $O L$ cells (Supplementary Figure 1B).

TRAF-MSCs exhibited similar characteristics to AF-MSCs. To obtain further insight into the changes in the gene expression profile during transdifferentiation, we performed a comparative analysis for the pluripotency markers Oct-4, Sox-2 and Nanog in TRAF-MSCs, AF-MSCs, $\mathrm{AL}$ and $\mathrm{HL}$ cells by Real-time PCR analysis. AF-MSCs and TRAF-MSCs exhibited higher levels of expression for Oct-4, Sox-2 and Nanog compared with $\mathrm{AL}$ and $\mathrm{HL}$ cells, documenting the primitive phenotype of the former cells (Figure 5a). In addition, the lysosomal activity and mitochondrial mass of TRAF-MSCs determined by LysoTracker and MitoTracker fluorescent dyes, respectively, (mitochondria/ lysosomal fluorescence ratio, $0.8 \pm 0.23$ ) was similar to AF-MSCs (mitochondria/lysosomal fluorescence ratio, $0.78 \pm 0.16)$. However, $\mathrm{AL}$ cells and $\mathrm{HL}$ cells exhibited significantly lower ratios of mitochondria/lysosomal fluorescence $0.52 \pm 0.04$ and $0.31 \pm 0.04$, respectively (Figure $5 \mathrm{~b}$ ii). Moreover, semiquantitative $\mathrm{PCR}$ analysis revealed that TRAF-MSCs did not express any of the adipogenic markers, such as fatty acid-binding protein 4 (FABP4), PPAR $\gamma$ and LPL (Figure $5 \mathrm{c}$ i). The hepatogenic marker tyrosine aminotransferase was expressed in similar levels in AF-MSCs and TRAF-MSCs ( $P=0.1087$, Student's $t$-test), which implies that later cell types have returned to a more primitive stem cell-like stage (Figure $5 \mathrm{c}$ ii). 
a

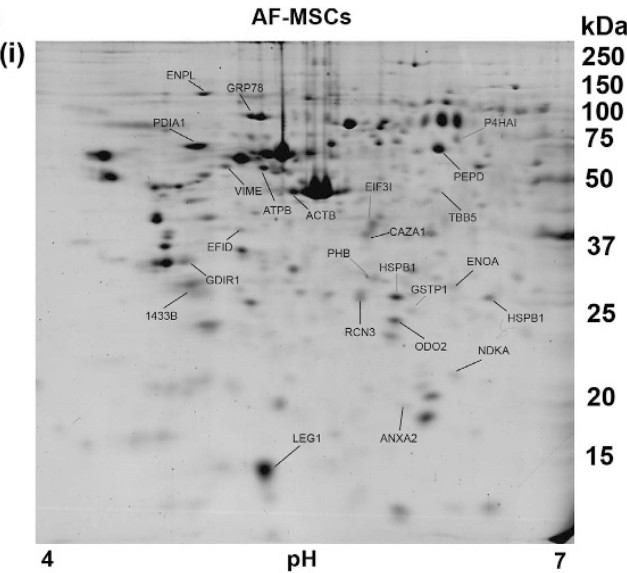

(ii)

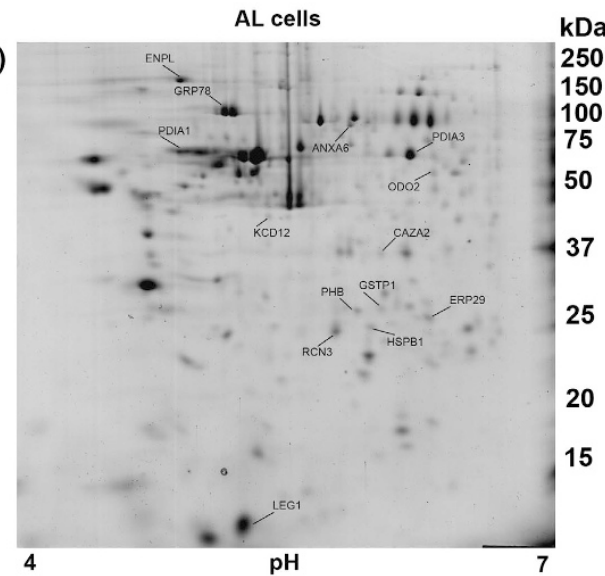

DAF-MSCs

(iii)

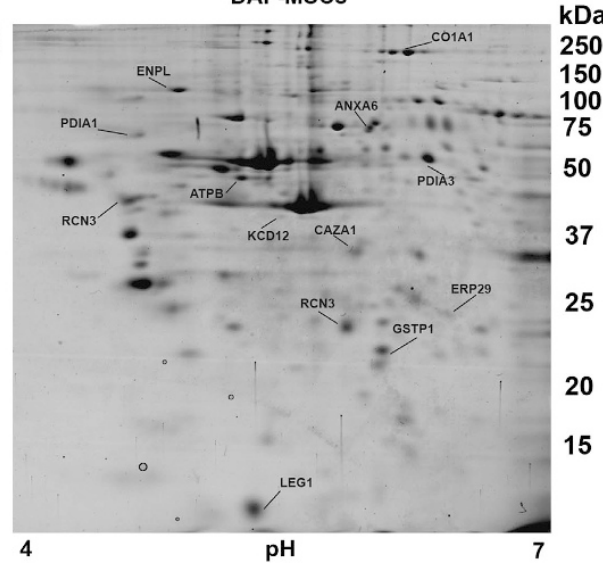

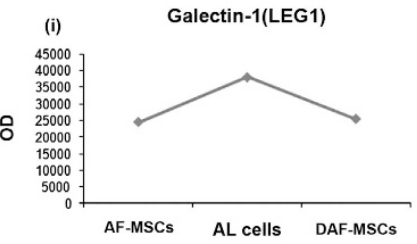
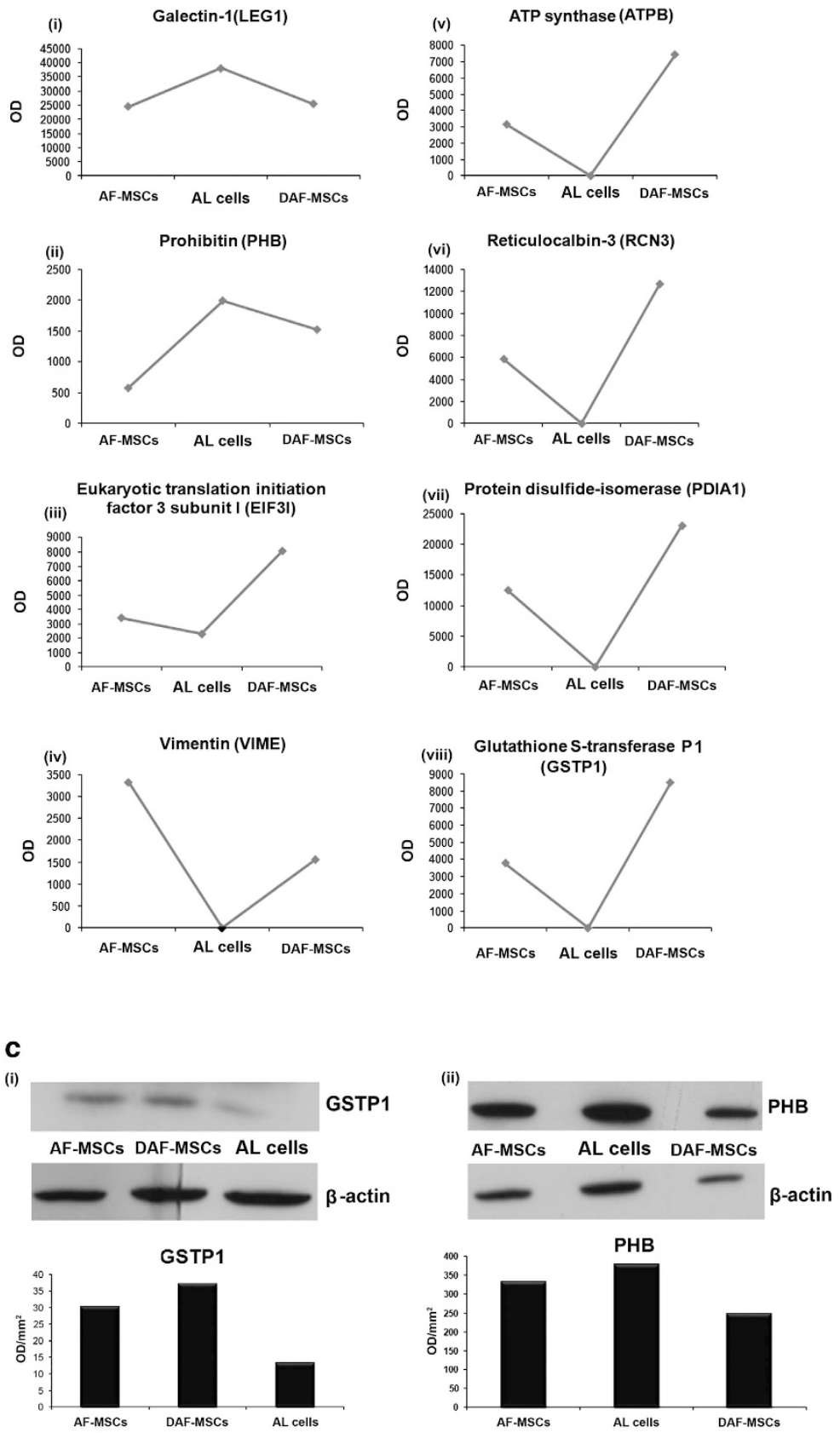

Figure 32 2DE analysis of AF-MSCs, AL cells and DAF-MSCs. (a) Representative 2DE gel image of proteins identified from (i) AF-MSCs, (ii) AL cells and (iii) DAF-MSCs. The protein spots in each population are indicated with their abbreviated names listed in Supplementary Table 2. (b) Comparative expression analysis for: (i) LEG-1, (ii) PHB, (iii) EIF3I 3 subunit, (iv) VIME, (v) ATPB subunit- $\beta$, (vi) RCN3, (vii) PDIA1 and (viii) GSTP1. (c) Western blot analysis in cell extracts from AF-MSCs, AL cells and DAF-MSCs for (i) GSTP1 $\mathrm{P} 1$ and (ii) PHB expression. Protein bands of 32 and $26 \mathrm{kDa}$ corresponding to the two above proteins were detected. $\beta$-actin was used as positive control for equal loading

AF-MSCs, DAF-MSCs and TRAF-MSCs exhibit similar clonogenic potential and proteome profile. AF-, DAFand TRAF-MSCs shared similarities in clonogenic potential, as determined by 'colony-forming' unit fibroblast (CFU-F) assay (Figure 6a). In addition, all three cell types expressed Oct-4, Sox-2 and Nanog at protein level, as demonstrated by immunofluorescent staining (Figure 6b). Moreover, we performed a proteomic analysis of the AF-, DAF- and TRAF-MSCs, identifying differentially expressed proteins by 2DE gel and MS (Supplementary Figure 3). A comparison of the expression levels of the respective proteins was established using PDQuest 8 software (Bio Rad Laboratories Inc, Hercules, CA, USA). Proteins differentially expressed $>1.5$-fold $(P<0.05$, Student's $t$-test $)$ in the three populations, 


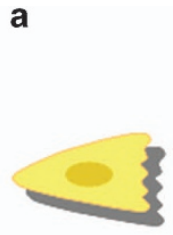

AF-MSCs

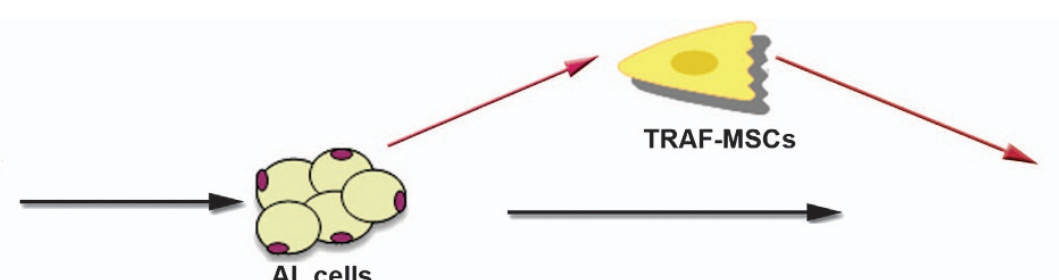

AL cells

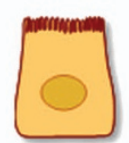

HL cells
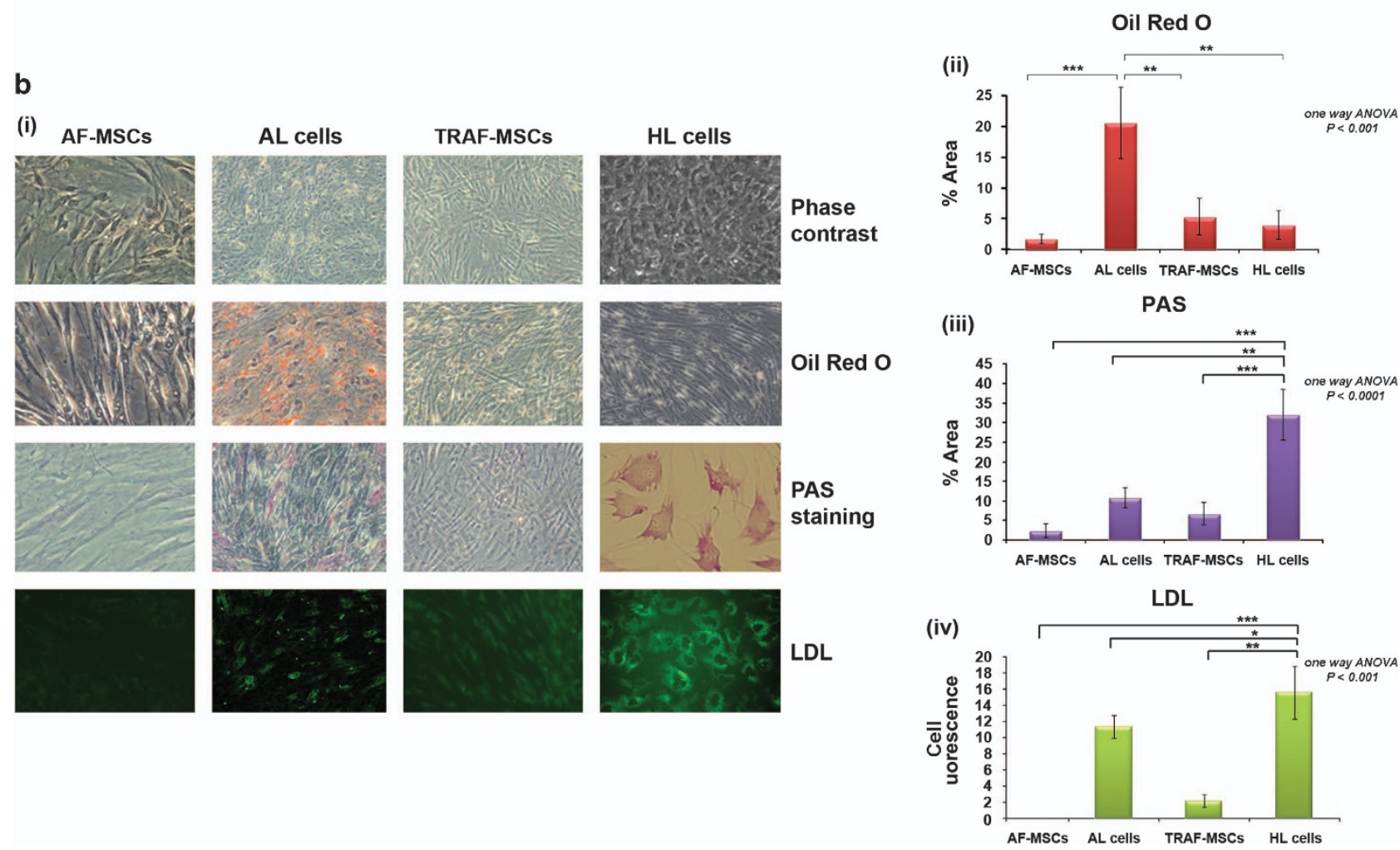

Figure 4 Transdifferentiation of AL cells into HL cells. (a) AF-MSCs were induced into AL cells, for 2 weeks in adipogenic medium, and then were transdifferetiated into HL cells through a dedifferentiation step (TRAF-MSCs). (b) (i) Morphology of AF-MSCs, AL cells, TRAF-MSCs and HL cells, detection of lipid droplets and glycogen storage and LDL uptake, (ii) quantitation analysis for Oil Red O staining, (iii) PAS staining and (iv) LDL uptake. Data are presented as the mean \pm S.D., and were statistically analyzed by one-way ANOVA. Additional statistical analysis pairwise was performed using Student's $t$ test, $\left({ }^{\star \star} P<0.01,{ }^{* \star *} P<0.001\right)$

are described in detail in Supplementary Table 2. Specifically, the metabolic protein Xaa-Pro dipeptidase (PEPD) ${ }^{28}$ was found to be expressed in high level (5- to 4-fold expression difference) in AF-MSCs compared with TRAF-MSCs. Moreover, Rho-GDP-dissociation inhibitor 1 (GDIR1) was expressed in higher levels in DAF-MSCs and TRAF-MSCs compared with AF-MSCs. GDIR1 is reported to participate in the process of dedifferentiation of smooth muscle cells. ${ }^{29}$ In addition, TRAF-MSCs expressed in high levels the elongation factor 1-delta (EF1D) and peroxiredoxin-4 (PRDX4), both related to differentiation of MSCs. ${ }^{30,31}$

To define the differences in the profile of the molecular mediators secreted by AF-, DAF- and TRAF-MSCs, we examined the conditioned medium (CM) derived from AF-MSCs and directly compared this with DAF-MSC-CM and TRAF-MSC-CM, respectively, using proteome profiler human arrays (Figure 7a). The array analysis allowed the identification of 20 secreted growth factors for AF-MSCs, 16 for DAF-MSCs and 25 for TRAF-MSCs (Supplementary Figure 4).
According to the results obtained, all three cell types secreted growth factors, which facilitate angiogenesis, such as angiogenin, ${ }^{32}$ angiopoietin- $1,{ }^{33}$ endostatin/collagen $\mathrm{XVIII},{ }^{34}$ transforming growth factor- $\beta \quad 1 \quad(\mathrm{TGF} \beta 1)^{35}$ and vascular endothelial growth factor (VEGF) ${ }^{19,36}$ (Figure $7 b$ i). The Fibroblast growth factor-7 (FGF-7), ${ }^{37}$ Serpin E1, Serpin $\mathrm{F}^{38}$ and urinary plasminogen activator (UPA) ${ }^{39}$ proteins, responsible for the tissue repair and regeneration, were also detected in CM derived from AF-, DAF- and TRAF-MSCs (Figure $7 \mathrm{~b}$ ii). More importantly, these cells also secreted Interleukin-8 (IL-8) ${ }^{19,40}$ and the matrix metalloproteinase-9 (MMP-9) ${ }^{41}$ which have been suggested as major regulators of the mobilization of MSCs (Figure 7b iii). Additionally, AF-, DAF- and TRAF-MSCs secreted factors that have a key role in cell differentiation, such as insulin-like growth factor-binding protein 2 (IGFBP-2), ${ }^{42}$ IGFBP- $3^{43}$ and the tissue inhibitor of metalloproteinase 1 (TIMP-1) ${ }^{44}$ (Figure $7 \mathrm{~b}$ iv, v).

More interestingly, TRAF-MSCs showed higher expression of TGF $\beta 1$ and IGFBP-2 molecules, both involved in signaling pathways related to multiple biological processes, including 

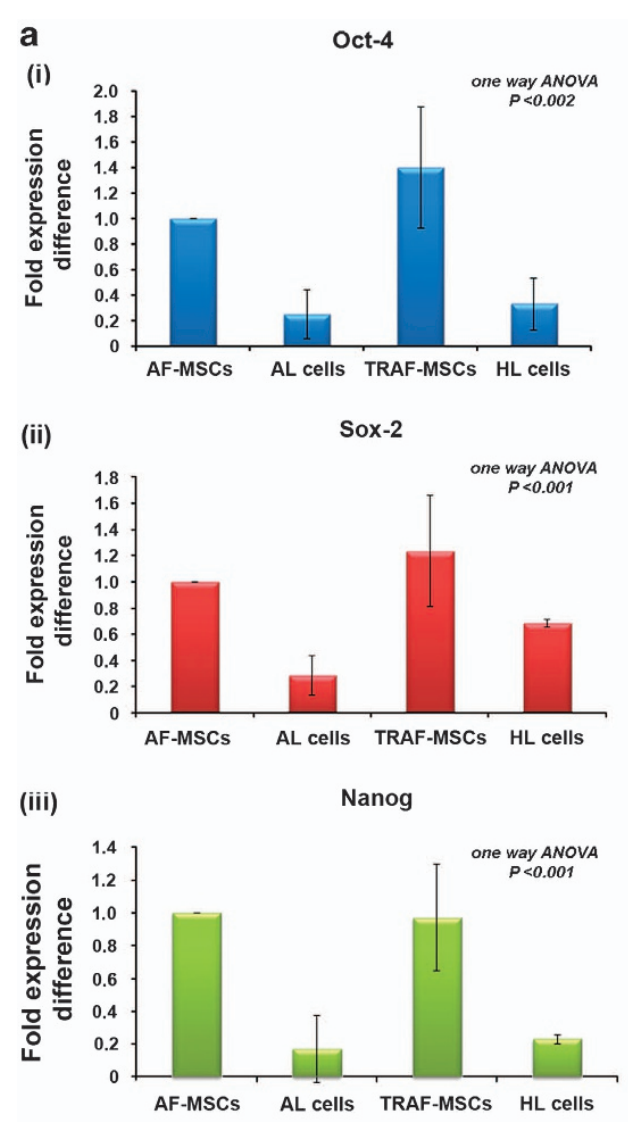

C

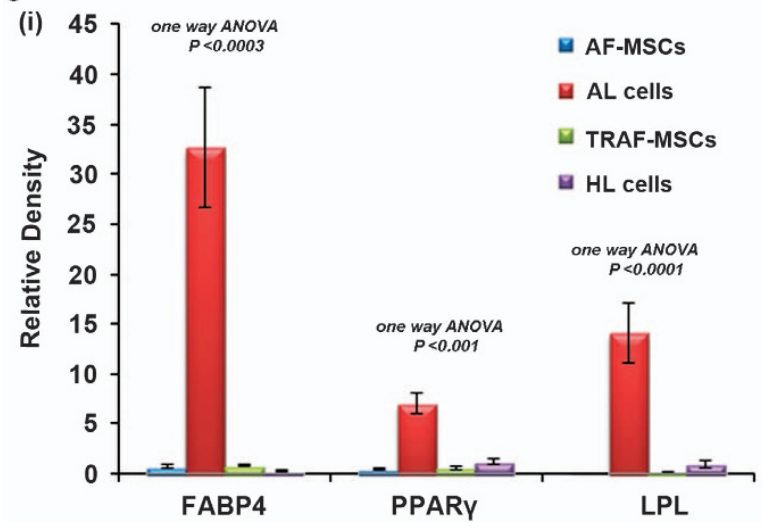

(ii) b
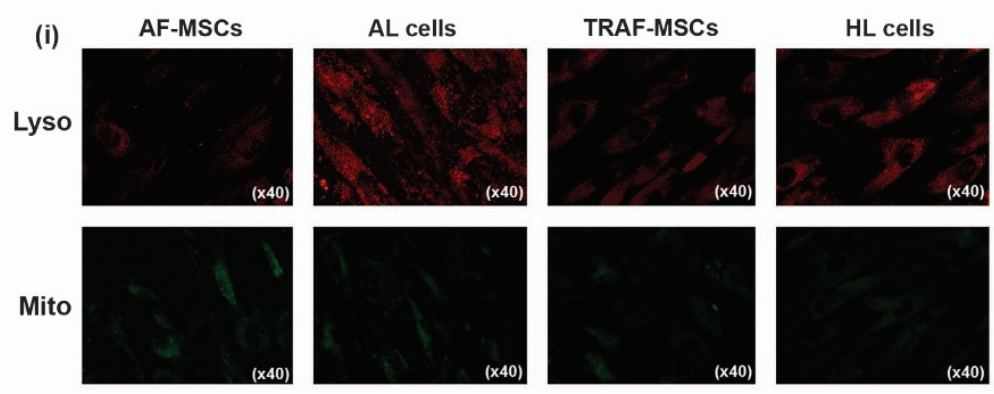

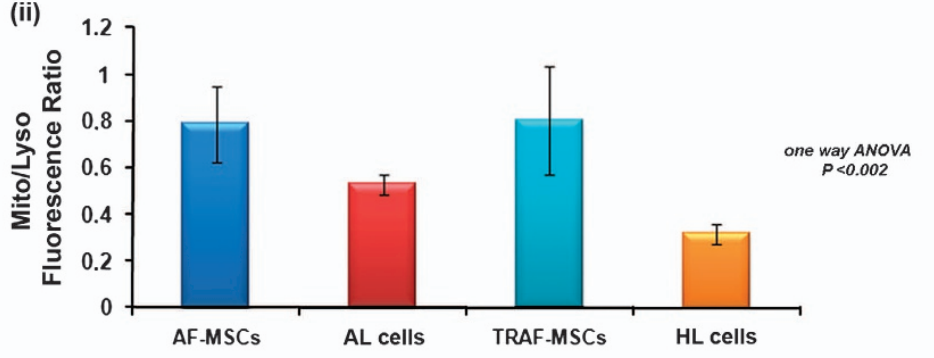

(ii)

\section{Tyrosine aminotransferase}

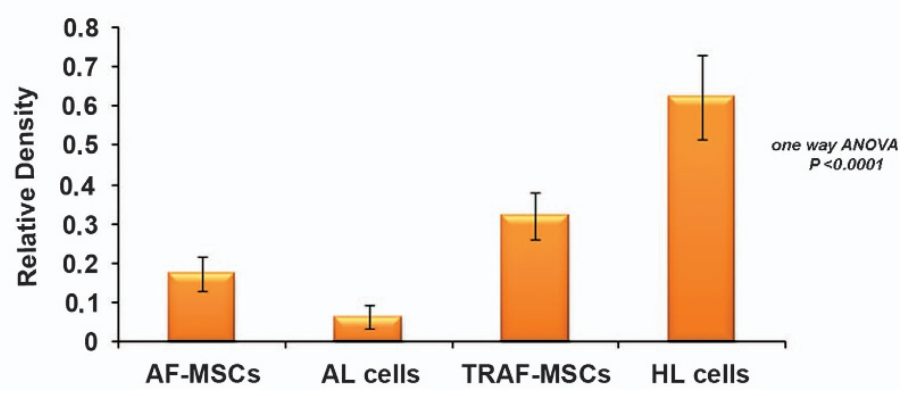

Figure 5 Stem cell marker expression and metabolic activity of AF-MSCs, AL cells, TRAF-MSCs and HL cells. (a) Real-time PCR expression analysis for (i) Oct4, (ii) Sox-2 and (iii) Nanog in AF-MSCs, AL, TRAF-MSCs and HL cells. Data are presented as the mean \pm S.D. of three independent experiments, and the statistical significance was defined by one-way ANOVA. (b) (i) Microscope photos of labeled lysosomes (red) and mitochondria (green) on AF-MSCs, AL cells, TRAF-MSCs and HL cells. (ii) Ratios of MitoTracker/LysoTracker for the four cell types. Data are presented as the mean \pm S.D. of 10 images per cell category, and were statistically analyzed by one-way ANOVA. (c) Semiquantitative PCR analysis for the expression of (i) the adipogenic markers, FABP4, PPAR $\gamma$ and LPL and (ii) the hepatogenic marker, tryrosine aminotransferase in AF-MSCs, AL, TRAF-MSCs and HL cells. Tyrosine aminotransferase was expressed in similar levels in AF-MSCs and TRAF-MSCs $(0.05<P<0.1$, Student's t-test). Data are presented as the mean \pm S.D. of three independent experiments and the statistical significance was defined by one-way ANOVA

cell proliferation, differentiation and also transdifferentiation through the regulation of specific genes. ${ }^{45,46}$ Moreover, TRAF-MSCs secreted Serpin E1, IL-8 and UPA in higher levels, factors that are increased during the process of transdifferentiation ${ }^{47-49}$ and are thought to be stimulated by the TGF $\beta$ protein $^{50}$ (Figure 7b).

It should be mentioned that a number of proteins identified by proteomic analyses together with the detected secreted molecules may participate in signaling pathways, by regulating differentiation, angiogenesis and mobilization of MSCs. In this respect, TGF $\beta$, secreted by both AF- and TRAFMSCs, is reported to stimulate VIME, a protein detected in our proteomic analysis, regulating in this way osteoblast differentiation ${ }^{51}$ and TGF $\beta$-mediated fibroblastic transdifferentiation. ${ }^{52}$ Similarly, HSPB1 was found in high levels in AF-, DAF- and TRAF-MSCs, and is reported to 
a (i)

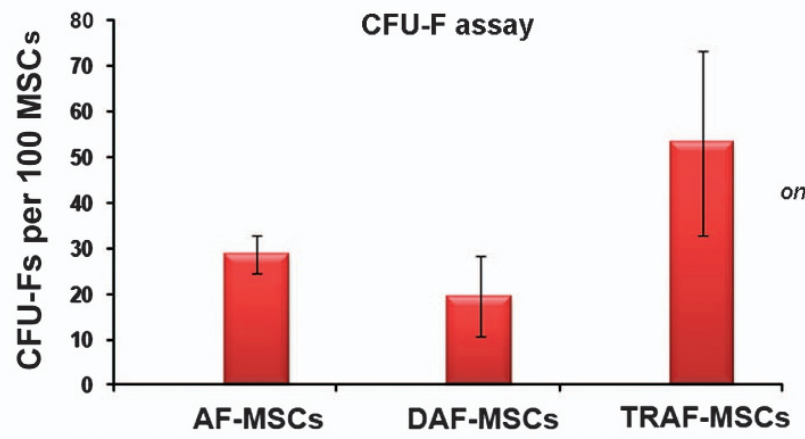

(ii)

\section{b}
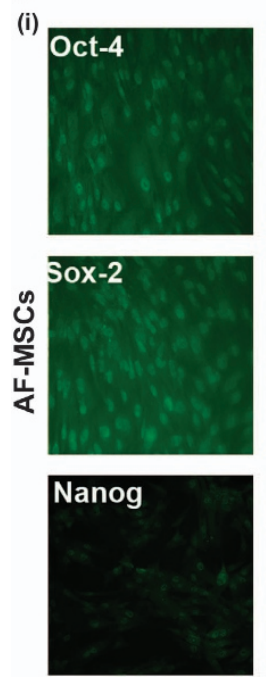
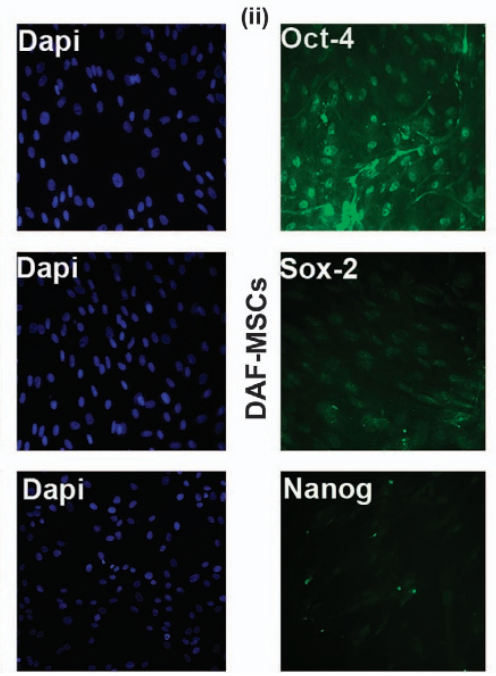
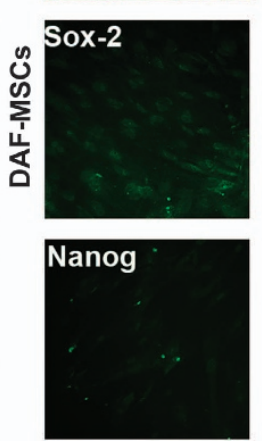
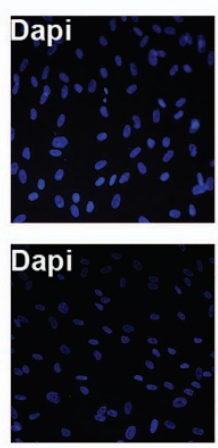

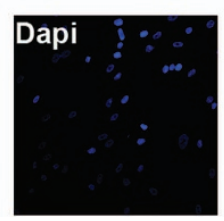

one way ANOVA

$P<0.01$

Figure 6 Clonogenic potential and expression of stem cell markers for AF-, DAF- and TRAF-MSCs. (a) (i, ii) The clonogenic potential of AF-, DAF- and TRAF-MSCs was determined by CFU-F assay. The mean numbers \pm S.D. of CFU-F per 100 cells formed in a 14-day clonogenic assay are presented, $P$ value $<0.0107$, one-way ANOVA. (b) Immunofluorescent staining for Oct-4, Sox-2 and Nanog in (i) AF-MSCs, (ii) DAF-MSCs and (iii) TRAF-MSCs. DAPI solution was used for nuclear staining. Original magnifications, $\times 20$

interact with the VEGF and to regulate angiogenesis. ${ }^{53} \mathrm{LEG}-1$ was also detected in the three cell types tested and is involved in the modulation of JAK/STAT pathway, which is implicated in HGF and EGF signaling. ${ }^{54}$

\section{Discussion}

Previous studies have shown that adult stem cells, like MSCs, are able to transdifferentiate from a specific developmental lineage into another cell type of a different lineage. ${ }^{11,14}$ Their cell fate is determined by the expression of several cytokines, growth factors, adhesion molecules and extracellular matrix components. ${ }^{55}$ In particular, Tuan and colleagues ${ }^{11}$ demonstrated that human MSCs derived from the BM (BMMSCs), after being differentiated into osteocytes, chondocytes and adipocytes, can transdifferentiate into other cell types under appropriate culture conditions. This study proposed that differentiated BM-MSCs dedifferentiate into a primitive stem cell-like stage before transdifferentiation, likely through genetic reprogramming. To support their findings, the same group showed that BM-MSCs, induced to osteogenesis, adipogenesis or chondrogenesis, could dedifferentiate into a primitive stem cell-like population, upon the withdrawal of the stimulating culture medium. ${ }^{14}$

More recently, human second-trimester AF has been characterized as a very promising fetal source of MSCs. ${ }^{4-7,13}$ 
a

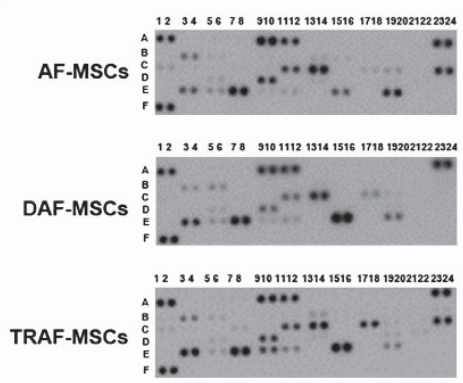

(iii) Mobilization of MSCs

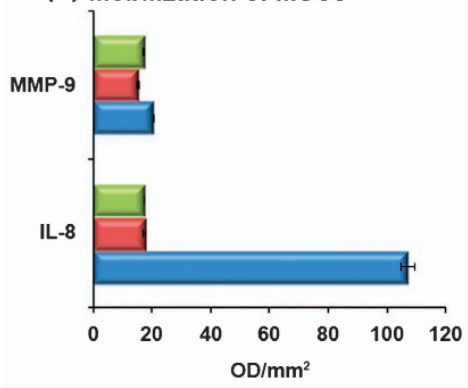

b

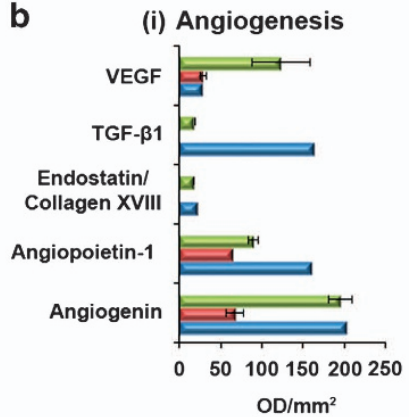

(iv) Cell differentiation

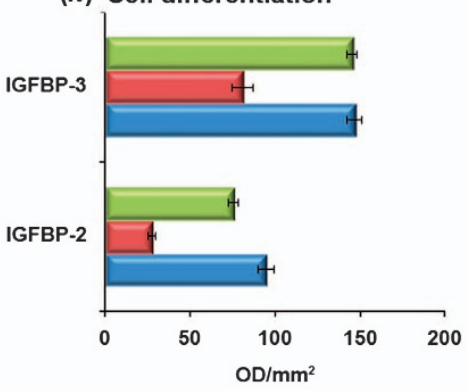

(ii) Tissue repair

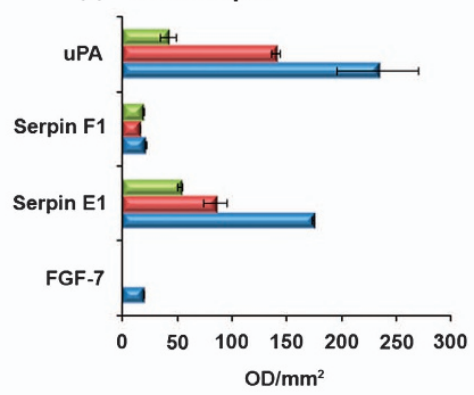

(v) Apoptosis

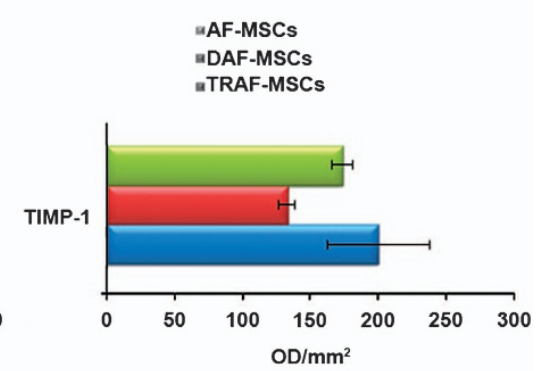

c

\begin{tabular}{|c|c|c|c|c|c|c|c|c|c|c|c|c|}
\hline & 1,2 & 3,4 & 5,6 & 7,8 & 9,10 & 11,12 & 13,14 & 15,16 & 17,18 & 19,20 & 21,22 & 23,24 \\
\hline & POS & & Activin A & ADAMTS-1 & Angiogenin & Angiopoietin-1 & Angiopoietin-2 & \begin{tabular}{|l} 
Angiostatin/ \\
Plasminogen
\end{tabular} & Amphiregulin & Artemin & & \\
\hline & $\begin{array}{l}\text { Ooagulation } \\
\text { factor } \\
\text { III }\end{array}$ & CXCL16 & DPPIV & EGF & EG-VEGF & Endoglin & \begin{tabular}{|} 
Endostatin/ \\
Collagen XVIII
\end{tabular} & Endothelin-1 & Endothelin-1 & FGF acidic & FGF-4 & FGF-7 \\
\hline c & GDNF & GM-CSF & HB-EGF & HGF & IGFBP-1 & IGFBP-2 & IGFBP-3 & IL-1 $\beta$ & |L-8 & LAP (TGF- $\beta 1$ ) & Leptin & MCP-1a \\
\hline D & MIP-1a & MMP-8 & MMP.9 & NRG1- $\beta 1$ & Pentraxin 3 & PD-ECGF & PDGF-AA & $\begin{array}{l}\text { PDGF-ABI } \\
\text { PDGF-BB }\end{array}$ & Persephin & Platelet factor 4 & PIGF & Prolactin \\
\hline E & Serpin B5 & Serpin E1 & Serpin F1 & TIMP-1 & TIMP-4 & \begin{tabular}{|} 
Thrombo- \\
spondin-1
\end{tabular} & $\begin{array}{l}\text { Thrombo- } \\
\text { spondin-2 }\end{array}$ & UPA & Vasohibin & VEGF & VEGF-C & \\
\hline FF & POS & & & & & & & & & & & NEG \\
\hline
\end{tabular}

Figure 7 AF-MSC, DAF-MSC and TRAF-MSC secretome analyses. (a) Representative proteome profile image for growth factors secreted in CM of AF-MSCs, DAFMSCs and TRAF-MSCs. (b) Expression levels of growth factors related to (i) angiogenesis, (ii) tissue repair, (iii) mobilization of MSCs, (iv) cell differentiation and (v) apoptosis in CM of AF-MSCs, DAF-MSCs and TRAF-MSCs. Data presented as mean \pm S.D. of at least three independent experiments. (c) Corresponding names of each molecule within the array summarized in tabular form

In our previous studies, we have successfully isolated and expanded karyotypically normal MSCs from AF and performed a systematic phenotypic, molecular and proteomic analysis. The main characteristics of AF-MSCs included the high number of isolated cells and their rapid expansion in vitro compared with MSCs from adult sources such as BM-MSCs. ${ }^{7}$ More importantly, these cells when exposed to appropriate differentiation culture medium in vitro, showed a multilineage differentiation potential and ability to overcome the mesodermal commitment by differentiating into hepatocytes. ${ }^{6,7,12}$ Furthermore, AF-MSCs and their hepatic progenitors induced liver repair and support liver function by cell transplantation into acute hepatic failure animal model. $., 7,12$

In the present study, we used a well-defined cell culture system to determine whether differentiated AF-MSCs were able to maintain their plasticity. Specifically, we have shown that AF-MSCs could successfully differentiate into $A L$ cells and then these cells, under certain culture conditions, were able to dedifferentiate and acquire a more primitive phenotype. More particularly, we proved that DAF-MSCs expressed the stem cell markers SSEA-4, Oct-4, Sox-2 and Nanog in high levels, documenting a similar gene expression profile to AF-MSCs. Moreover, we studied the lysosomal activity changes in AF-MSCs during the processes of differentiation and dedifferentiation. Additionally, we performed a comparative proteomic analysis of AF-MSCs, AL cells and DAF-MSCs, using 2DE and MS analysis. In particular, 31 proteins were found to be differentially expressed among the three cell populations and more interestingly, some of the proteins, were expressed at the same levels in AF-MSCs and DAFMSCs compared with $\mathrm{AL}$ cells. These proteins included VIME, which regulates integrin function, migration and cell signaling, ${ }^{56}$ LEG-1, which supports cell differentiation and is an important stem cell regulatory molecule, ${ }^{57}$ and $\mathrm{PHB}$, which promotes cell proliferation and development. ${ }^{23}$

Moreover, according to our data, precommitted AF-MSCs to adipogenesis could transdifferentiate into hepatocytes in response to specific extracellular signals. Thus, the important question raised was whether differentiated cells into mesodermal lineage ( $A L$ cells) could transdifferentiate into endoderm-derived cells (HL cells) directly or through the process of dedifferentiation. For this reason, AL cells were induced to hepatogenesis for 21 days before hepatic culture condition initiation. On the 4th day of transdifferentiation, cells exhibited similar phenotypic characteristics to AF-MSCs and were termed as TRAF-MSCs. Interestingly enough, TRAF-MSCs expressed 
the pluripotency markers Oct-4, Sox-2 and Nanog, implying a similar gene profile to undifferentiated AF-MSCs, and also exhibited the same lysosomal activity to AF-MSCs and maintained their differentiation potential.

In addition, we observed that AF-, DAF- and TRAF-MSCs displayed similar clonogenic potential and proteome profile as determined by 2DE gel and MS analysis. We further examined the secretome of AF-, DAF- and TRAF-MSCs by using a proteome profile human array. We have concluded that all three cell types were able to produce molecules, which were related to angiogenesis, tissue repair and cell differentiation, documenting the significance of the plasticity of these cells in tissue regeneration. Moreover, TRAF-MSCs expressed a number of proteins related to the TGF $\beta 1$ - and IGF signaling pathways in higher levels compared with AF-MSCs. However, further studies are needed in order to identify the molecular mediators that participate in the process of transdifferentiation.

In conclusion, our study has demonstrated that MSCs derived from AF are capable to dedifferentiate into a more primitive stem cell type and to transdifferentiate from mesoderm- to endoderm-derived cells in response to extrinsic factors, likely through an initial dedifferentiation step, retaining their multipotentiality. These processes share many similarities with reprogramming. Similar to the first report of Takahashi and Yamanaka, ${ }^{58}$ many different cell types have been reprogrammed by a combination of exogenous transcription factors. However, several reports have suggested that dedifferentiation does not require transcription factors' introduction, and could be achieved through specific culture conditions. ${ }^{59}$

More particularly, a recent study has shown that in vitro culture could convert epiblast stem cells or progenitor cells from mouse testis into embryonic stem cell-like cells without the use of exogenous reprogramming factors, but by specified in vitro culture conditions. ${ }^{60}$ More recently, Moschidou et al. ${ }^{15}$ showed that stem cells derived from AF can be fully reprogrammed to pluripotency without genetic manipulation, just in the presence of human embryonic stem cell medium supplemented with the valproic acid, a histone deacetylase inhibitor.

Dedifferentiation and transdifferentiation, under specific culture conditions, could be valuable tools in cell therapy, being able to generate tissue-specific cells in vitro that can be possibly used for clinical applications in the future. ${ }^{16}$ The present study may support the use of AF-MSCs in future cell-based therapies. However, the efficient establishment of reprogramming is a major issue and requires a thorough study.

\footnotetext{
Materials and Methods

Isolation and culture expansion of AF-MSCs. AF-MSCs were isolated from six AF samples, collected during scheduled amniocentesis between the 15th and 18 th weeks of gestation, as described previously. ${ }^{6,7}$ All second-trimester AF samples were obtained following a written informed consent, approved by the Ethical Committee of the Alexandra Hospital, Athens and the Bioethics Committee of the University of Athens, School of Medicine, Athens. Following collection, $10-15 \mathrm{ml}$ from each sample was centrifuged at 1300 r.p.m. for $10 \mathrm{~min}$. The pellet was resuspended in Dulbecco's modified Eagle's medium (DMEM; Sigma-Aldrich Ltd., Gillingham, Dorset, UK) supplemented with $20 \%$ (v/v) fetal bovine serum (FBS; Gibco-BRL, Paisley, Scotland, UK) in a $25-\mathrm{cm}^{2}$ tissue culture-treated flask and incubated at $37^{\circ} \mathrm{C}$ in a $5 \%(\mathrm{v} / \mathrm{v})$ humidified $\mathrm{CO}_{2}$ chamber for $\sim 20$ days, when the first colonies appeared. Mechanical isolation was performed to isolate the spindleshaped (SS) AF-MSCs, according to methods described in our previous studies. ${ }^{6}$ Cells were used for differentiation at passage $3-5$.
}

Differentiation, dedifferentiation and transdifferentiation of AF-MSCs. Differentiation of AF-MSCs, DAF-MSCs or TRAF-MSCs into AL, osteocyte-like (OL) and $\mathrm{HL}$ cells was induced. More particularly, to induce adipogenesis, AF-MSCs or DAF-MSCs, at $1.0-1.3 \times 10^{4} / \mathrm{cm}^{2}$ density were treated with adipogenic medium, as previously described for 1,2 or 3 weeks. ${ }^{6,7}$ For hepatogenic differentiation, AF-MSCs or DAF-MSCs, at the same density, were serum-deprived for 2 days. Hepatogenesis was induced by treating AF-MSCs or DAF-MSCs with differentiation medium, as previously described. ${ }^{6,7,12}$ To induce dedifferentiation process, after 2 weeks in culture the adipogenic medium was removed from the AL cells and it was replaced by AF-MSC medium for 1,2 and 3 weeks. After 2 weeks in AF-MSC medium, cells exhibited a phenotype similar to that of AF-MSCs, and were termed as DAF-MSCs (dedifferentiated AF-MSCs). For transdifferentiation, AF-MSCs were cultured in adipogenic medium for 2 weeks, and then were cultured in hepatogenic medium for further 21 days. After 4 days in culture, cells regained a more primitive phenotype similar to AF-MSCs, and were termed as TRAF-MSCs. To induce osteogenesis, DAF-MSCs and TRAF-MSCs were cultured in osteogenic medium for 3 weeks, as described previously. ${ }^{6,7}$

Differentiation functional assays. Adipogenesis was determined by analyzing the lipid formation performing Oil Red O staining (Sigma-Aldrich Ltd.). Staining was assessed by bright-field microscopy and quantified by the Image J 1.38x software (Millersville University, Millersville, PA, USA). Quantitative destaining of Oil Red $\mathrm{O}$ staining was performed by adding $1 \mathrm{ml}$ of isopropyl alcohol (Sigma-Aldrich Ltd.) and measuring the absorbance at $500 \mathrm{~nm}$ using a spectrophotometer. The percentage of Oil Red $O$ staining was calculated using the formula: $[(O D$ day $x-O D$ day 0$) / O D$ day $0 \times 100)]$. Three independent experiments were performed, each including three replicates, and the mean \pm S.D. of each experiment was calculated.

To analyze hepatogenic differentiation, the cells were assayed for the glycogen storage, by using a Periodic Acid-Schiff staining system (Sigma-Aldrich). Additionally, LDL uptake was estimated. Briefly, cells were incubated overnight at $37^{\circ} \mathrm{C}$ in a $5 \%(\mathrm{v})$ v) humidified $\mathrm{CO}_{2}$ chamber in DMEM-HG supplemented with $2.5 \mu \mathrm{g} / \mathrm{ml}$ Alexa Fluor 488 ACLDL (Molecular Probes Inc.) in the absence of serum. The next day, cells were examined under a Leica CTR-MIC fluorescent microscope (Buffalo Grove, IL, USA) and cell fluorescence was analyzed with the Image J 1.38x software.

Osteogenesis was determined by Alizarin Red S (Sigma-Aldrich Ltd.) staining. Solubilized Alizarin red $\mathrm{S}$ was destained by adding 1-ml 10\% cetylpyridinium chloride (Sigma-Aldrich Ltd.), followed by measurement of the absorbance at $570 \mathrm{~nm}$ using a spectrophotometer. The percentage of Alizarin red S staining was calculated using the formula: [(OD day $x-O D$ day 0$) / O D$ day $0 \times 100)]$. Three independent experiments were performed, each including three replicates, and the mean \pm S.D. of each experiment was calculated.

'Colony-forming' unit fibroblast assay. A CFU-F assay was used for the evaluation of the clonogenic potential of AF-MSCs (passage 5-7), DAF-MSCs and TRAF-MSCs. CFU-F assay was performed by plating cells at three clonal densities (70, 140 and 280 cells) in 60-mm plates for 14 days. CFU-Fs were quantified using Giemsa staining. The number of CFU-Fs was estimated per 100 MSCs initially plated, on the basis of the linear regression analysis obtained from the three different initial cell concentrations.

Antibodies and flow cytometry analysis. AF-MSCs, DAF-MSCs and AL cells were examined for the expression of SSEA-4 embryonic stem-cell marker by flow cytometry at different time points. The cells were stained with the antibody against SSEA-4 (Abcam, Cambridge, UK). As secondary antibody, the isothiocyanate (FITC)-conjugated goat anti-mouse immunoglobulin G (IgG; DAKO DakoCytomation Ltd, Cambridgeshire, UK) was used. Flow cytometry was conducted by using Beckman Coulter Cytomics FC 500 flow cytometer (Beckman Coulter Ltd, Palo Alto, CA, USA).

Immunofluorescent staining. For immunofluorescent staining, cells were permeabilized in $0.1 \%(\mathrm{v} / \mathrm{v})$ Triton-X (Sigma-Aldrich), supplemented with 5\% donkey serum (Sigma-Aldrich) for $30 \mathrm{~min}$ at room temperature after fixation in $4 \%$ paraformaldehyde (Sigma-Aldrich). Cells were then stained with rabbit anti-human Oct-3/4 (Santa Cruz Biotechnology Inc., Santa Cruz, CA, USA), rabbit anti-human Nanog (Abcam) or rabbit anti-human Sox-2 (Millipore, Hayward, CA, USA) antibodies. The latter reactions were developed with Alexa-488-conjugated goat anti-rabbit (Invitrogen Ltd., Grand Island, NY USA) secondary antibody. Slides were 
mounted with vectashield mounting medium (Vector Laboratories Inc., Burlingame, CA, USA) containing DAPI solution. Cells were visualized and photographed on a Leica CTR-MIC Fluorescent microscope.

Mitochondria and lysosomal fluorescent measurement. MitoTracker Green FM and LysoTracker Red (Molecular Probes Inc) were diluted in DMEM to the appropriate concentrations. Cells were incubated with prewarmed $\left(37^{\circ} \mathrm{C}\right)$ probe-containing medium for $1 \mathrm{~h}$ for LysoTracker Red and for $45 \mathrm{~min}$ for MitoTracker Green FM. Cells were visualized and photographed on a Leica CTRMIC Fluorescent microscope. All images were analyzed with Image $\mathrm{J} 1.38 \mathrm{x}$ software and the ratio of MitoTracker/LysoTracker was calculated for each sample (AF-MSCs, DAF-MSCs, TRAF-MSCs, AL cells and HL cells). Ten different areas from each image were used for statistical analysis.

RT-PCR procedure and semiquantitative PCR. Total RNA was extracted from the cells using the Tri Reagent (Sigma-Aldrich), according to the manufacturer's instructions. The mRNA was reverse transcribed to CDNA using the M-MLV Reverse Transcriptase-RNase H Minus kit (Promega, Madison, WI, USA). Subsequent PCR reactions were performed using CDNA, primer pairs and Go Taq Green master mix, (Promega). Semiquantitative PCR analysis was determined by using the Image $\mathrm{J} 1.38 \mathrm{x}$ software after normalization to the $\beta$-actin endogenous control. The primers used are listed in Supplementary Table 1.

Real-time quantitative PCR. Real-time quantitative PCR was conducted on an ABI Prism 7000 apparatus (Applied Biosystems, Foster City, CA, USA). Each CDNA sample was mixed with specific primer sets and PCR master mix (Applied Biosystems, No. 4312704). TaqMan real-time PCR was used for Oct-4, Sox-2 and Nanog gene expression analysis with primers and conditions designated by Assays on Demand, Gene Expression Products (Applied Biosystems Hs00742896_s1 (oct-4), Hs01053049_s1 9 (sox-2), Hs02387400_s1 (Nanog), respectively). Analysis was performed by using the ABI Prism 7700 SDS software (Applied Biosystems). The expression of Oct-4, Sox-2 and Nanog was normalized to GAPDH internal control (GAPDH, TaqMan pre-developed assay reagents, Applied Biosystems, No. 433764T). Data were analyzed by the relative quantification $(\Delta \Delta \mathrm{Ct})$ method, as described in our previous studies. ${ }^{6,7}$ Then, the relative level of Oct-4, Sox-2 or Nanog in AF-MSCs was compared with DAFMSCs, TRAF-MSCs, AL cells or HL cells by setting the AF-MSCs gene expression value to 1 and determining the fold change in expression against this value, using the following formula: $2^{-\Delta \Delta C t}$. Quantitative real-time PCR was carried out for PPAR $\gamma$, FABP4, Runx2, osteocalcin, tyrosine aminotransferase and AFP using SYBR Green PCR master mix, according to the manufacturer's protocol (Applied Biosystems) and appropriate primers (Invitrogen Ltd.). $\beta$-actin was used as the internal control. The primers used are listed in Supplementary Table 1.

Proteomic analysis. At least three biological samples of the four categories (AF-MSCs, AL cells, DAF-MSCs and TRAF-MSCs) were analyzed by 2DE in triplicates, as was described previously. ${ }^{7}$ The protein identification was based on specific criteria, which included a maximum allowed mass error of 25 p.p.m. and a minimum of five matching peptides. More particularly, a high percentage of the proteins were identified based on 10 matches and the probability of a false identity was usually lower than $10^{-5}$. Finally, the functional properties of the identified proteins were analyzed by the Swiss-Prot (http://www.expasy.org/sprot) and Human Protein Reference Databases (http://www.hprd.org). Protein expression was calculated (in p.p.m.) by using the PD Quest 8 software package (Bio Rad Laboratories Inc.), and is shown as a ratio of the intensity of protein spots in AFMSCs to AL cells, AF-MSCs to DAF-MSCs, DAF-MSCs to AL cells, AF-MSCs to TRAF-MSCs or DAF-MSCs to TRAF-MSCs. Comparison of the expression level of the various protein spots was conducted by the use of Student's $t$-test. In all cases, $P<0.05$ was considered statistically significant.

Western blot. Total protein extracts of AF-MSCs, AL cells and DAF-MSCs were separated by $10 \%$ SDS-PAGE under reducing conditions and electroblotted to Hybond-ECL NC membrane (Amersham Biosciences, Uppsala, Sweden). After blocking, membranes were incubated overnight at $4{ }^{\circ} \mathrm{C}$ with the primary antibodies: mouse anti-human PHB (Santa Cruz Biotechnology Inc.) and mouse anti-human GSTP1 (Santa Cruz Biotechnology Inc.). Mouse anti-human $\beta$-actin antibody (Sigma Aldrich, Ltd.) was used as a control of equal loading. Membranes were then incubated with anti-mouse HRP-conjugated secondary antibody (Santa Cruz Biotechnology Inc.) and developed by ECL (PerkinElmer, Waltham, MA,
USA) detection system. Films were scanned and images were analyzed using Quantity One software 4.4.1 (Bio Rad Laboratories Inc.).

Proteome array of AF-MSCs, DAF-MSCs and TRAF-MSCs. For the preparation of the CM, $1 \times 10^{6}$ AF-, DAF- or TRAF-MSCs were cultured until $80 \%$ confluent in their respective media, and then the media were replaced with DMEM containing $0.5 \%$ (v/v) FBS to prevent protein aggregation. The cells were cultured for further $24 \mathrm{~h}$ and the CM was collected and concentrated $\sim 10$-fold using ultra filtration units with a 3-kDa cut-off (Millipore, Bedford, MA, USA). The analysis for specific proteins was performed using proteome profiler human arrays (Catalog No. ARY007, R\&D Systems Inc., Minneapolis, MN, USA), according to the manufacturer's instructions. Quantitation of the detected spots was performed using the Quantity One Software 4.4.1 (Bio Rad Laboratories Inc). Values were normalized to positive controls. Data are presented as the mean \pm S.D. for three independent experiments

Statistical analysis. Statistical analysis was performed using GraphPad Prism IV software (GraphPad Software Inc., La Jolla, CA, USA). One-way ANOVA (Kruskal-Wallis) was used to analyze the statistical significance of data from different experimental groups. In all cases, $P$ value of $<0.05$ was considered significant. Statistical analysis pairwise was performed using Student's $t$ test, $\left({ }^{*} P<0.05\right)$ when applicable.

\section{Conflict of Interest}

The authors declare no conflicts of interest.

Acknowledgements. This research has been co-financed by the European Union (European Social Fund - ESF) and Greek national funds through the Operational Program 'Education and Lifelong Learning' of the National Strategic Reference Framework (NSRF) - Research Funding Program: Heracleitus II. Investing in knowledge society through the European Social Fund and by Grant (supporting postdoctoral researchers) no. LS7-3902 from the Greek Secretariat of Research and Technology, which is co-financed by the European Social Fund and the Greek state.

\section{Authorship Contributions}

$D$ Zagoura and $O$ Trohatou contributed to the experimental design, experimental procedures, data analysis and manuscript writing; $V$ Bitsika contributed to the experimental procedures and manuscript reviewing; M Makridakis and A Vlahou conducted proteomic analysis and manuscript reviewing; $\mathrm{K}$ I Pappa provided the amniotic fluid samples; M G Roubelakis conceived and designed the experiments, performed data analysis and wrote the manuscript and N P Anagnou conceived the experiments and contributed reagents and materials.

1. Delorme B, Ringe J, Pontikoglou C, Gaillard J, Langonne A, Sensebe L et al. Specific lineage-priming of bone marrow mesenchymal stem cells provides the molecular framework for their plasticity. Stem Cells 2009; 27: 1142-1151.

2. English K, French A, Wood KJ. Mesenchymal stromal cells: facilitators of successful transplantation? Cell Stem Cell 2011; 7: 431-442.

3. Mosna F, Sensebe L, Krampera M. Human bone marrow and adipose tissue mesenchymal stem cells: a user's guide. Stem Cells Dev 2011; 19: 1449-1470.

4. De Coppi P, Bartsch G Jr., Siddiqui MM, Xu T, Santos CC, Perin L et al. Isolation of amniotic stem cell lines with potential for therapy. Nat Biotechnol 2007; 25: 100-106.

5. In tAnker PS, Scherjon SA, Kleijburg-van der Keur C, Noort WA, Claas FH, Willemze $\mathrm{R}$ et al. Amniotic fluid as a novel source of mesenchymal stem cells for therapeutic transplantation. Blood 2003; 102: 1548-1549.

6. Roubelakis MG, Bitsika V, Zagoura D, Trohatou O, Pappa KI, Makridakis M et al. In vitro and in vivo properties of distinct populations of amniotic fluid mesenchymal progenitor cells. J Cell Mol Med 2010; 15: 1896-1913.

7. Roubelakis MG, Pappa KI, Bitsika V, Zagoura D, Vlahou A, Papadaki HA et al. Molecular and proteomic characterization of human mesenchymal stem cells derived from amniotic fluid: comparison to bone marrow mesenchymal stem cells. Stem Cells Dev 2007; 16: 931-952.

8. Troyer DL, Weiss ML. Wharton's jelly-derived cells are a primitive stromal cell population. Stem Cells 2008; 26: 591-599.

9. Abdulrazzak H, Moschidou D, Jones G, Guillot PV. Biological characteristics of stem cells from foetal, cord blood and extraembryonic tissues. J R Soc Interface 2010; 7(Suppl 6): S689-S706. 
10. Morigi M, Rota C, Montemurro T, Montelatici E, Lo Cicero V, Imberti B et al. Life-sparing effect of human cord blood-mesenchymal stem cells in experimental acute kidney injury. Stem Cells 2011; 28: 513-522.

11. Song $L$, Tuan RS. Transdifferentiation potential of human mesenchymal stem cells derived from bone marrow. FASEB J 2004; 18: 980-982.

12. Zagoura DS, Roubelakis MG, Bitsika V, Trohatou O, Pappa KI, Kapelouzou A et al. Therapeutic potential of a distinct population of human amniotic fluid mesenchymal stem cells and their secreted molecules in mice with acute hepatic failure. Gut 2011; 61: 894-906.

13. Prusa AR, Marton E, Rosner M, Bernaschek G, Hengstschlager M. Oct-4-expressing cells in human amniotic fluid: a new source for stem cell research? Hum Reprod 2003; 18: 1489-1493.

14. Song L, Webb NE, Song Y, Tuan RS. Identification and functional analysis of candidate genes regulating mesenchymal stem cell self-renewal and multipotency. Stem Cells 2006; 24: 1707-1718.

15. Moschidou D, Mukherjee S, Blundell MP, Drews K, Jones GN, Abdulrazzak H et al. Valproic acid confers functional pluripotency to human amniotic fluid stem cells in a transgene-free approach. Mol Ther 2012; 20: 1953-1967.

16. Jopling C, Boue S, IzpisuaBelmonte JC. Dedifferentiation, transdifferentiation and reprogramming: three routes to regeneration. Nat Rev Mol Cell Biol 2011; 12: 79-89.

17. Odelberg SJ, Kollhoff A, Keating MT. Dedifferentiation of mammalian myotubes induced by msx1. Cell 2000; 103: 1099-1109.

18. Zhang Y, Li TS, Lee ST, Wawrowsky KA, Cheng K, Galang G et al. Dedifferentiation and proliferation of mammalian cardiomyocytes. PLOS One 2010; 5: e12559.

19. Bitsika V, Roubelakis MG, Zagoura D, Trohatou O, Makridakis M, Pappa Kl et al. Human amniotic fluid-derived mesenchymal stem cells as therapeutic vehicles: a novel approach for the treatment of bladder cancer. Stem Cells Dev 2011; 21: 1097-1111.

20. Reyes JM, Fermanian S, Yang F, Zhou SY, Herretes S, Murphy DB et al. Metabolic changes in mesenchymal stem cells in osteogenic medium measured by autofluorescence spectroscopy. Stem Cells 2006; 24: 1213-1217.

21. Krebiehl G, Ruckerbauer S, Burbulla LF, Kieper N, Maurer B, Waak J et al. Reduced basal autophagy and impaired mitochondrial dynamics due to loss of Parkinson's diseaseassociated protein DJ-1. PLoS One 2010; 5: e9367.

22. Camby I, Le Mercier M, Lefranc F, Kiss R. Galectin-1: a small protein with major functions. Glycobiology 2006; 16: 137R-157R.

23. Mishra S, Ande SR, Nyomba BL. The role of prohibitin in cell signaling. FEBS J 2010; 277 : 3937-3946.

24. Bommer UA, Lutsch G, Stahl J, Bielka H. Eukaryotic initiation factors elF-2 and elF-3: interactions, structure and localization in ribosomal initiation complexes. Biochimie 1991; 73: 1007-1019.

25. Ivaska J, Pallari HM, Nevo J, Eriksson JE. Novel functions of vimentin in cell adhesion, migration, and signaling. Exp Cell Res 2007; 313: 2050-2062.

26. Honore $\mathrm{B}$. The rapidly expanding CREC protein family: members, localization, function, and role in disease. Bioessays 2009; 31: 262-277.

27. Bulleid NJ. Protein disulfide-isomerase: role in biosynthesis of secretory proteins. Adv Protein Chem 1993; 44: 125-150

28. Chiravuri M, Lee H, Mathieu SL, Huber BT. Homodimerization via a leucine zipper motif is required for enzymatic activity of quiescent cell proline dipeptidase. J Biol Chem 2000; 275 26994-26999.

29. Doevendans PA, van Eys G. Smooth muscle cells on the move: the battle for actin. Cardiovasc Res 2002; 54: 499-502.

30. Maurer MH. Proteomic definitions of mesenchymal stem cells. Stem Cells Int 2011; 2011: 704256.

31. Roche S, Delorme B, Oostendorp RA, Barbet R, Caton D, Noel D et al. Comparative proteomic analysis of human mesenchymal and embryonic stem cells: towards the definition of a mesenchymal stem cell proteomic signature. Proteomics 2009; 9: 223-232.

32. Hsiao ST, Asgari A, Lokmic Z, Sinclair R, Dusting GJ, Lim SY et al. Comparative analysis of paracrine factor expression in human adult mesenchymal stem cells derived from bone marrow, adipose, and dermal tissue. Stem Cells Dev 2012; 21: 2189-2203.

33. Wu Y, Chen L, Scott PG, Tredget EE. Mesenchymal stem cells enhance wound healing through differentiation and angiogenesis. Stem Cells 2007; 25: 2648-2659.

34. Yin J, Kim JK, Moon JH, Beck S, Piao D, Jin X et al. hMSC-mediated concurrent delivery of endostatin and carboxylesterase to mouse xenografts suppresses glioma initiation and recurrence. Mol Ther 2011; 19: 1161-1169.

35. Luo $\mathrm{H}$, Zhang $\mathrm{Y}$, Zhang $\mathrm{Z}$, Jin $\mathrm{Y}$. The protection of MSCs from apoptosis in nerve regeneration by TGF beta1 through reducing inflammation and promoting VEGFdependent angiogenesis. Biomaterials 2012; 33: 4277-4287.

36. Carmeliet $\mathrm{P}$, Jain RK. Molecular mechanisms and clinical applications of angiogenesis. Nature 2011; 473: 298-307.

37. Braun S, auf dem Keller U, Steiling $H$, Werner S. Fibroblast growth factors in epithelial repair and cytoprotection. Philos Trans R Soc Lond B Biol Sci 2004; 359: 753-757.

38. Providence KM, Higgins SP, Mullen A, Battista A, Samarakoon R, Higgins CE et al. SERPINE1 (PAI-1) is deposited into keratinocyte migration 'trails' and required for optimal monolayer wound repair. Arch Dermatol Res 2008; 300: 303-310.
39. Vallabhaneni KC, Tkachuk S, Kiyan Y, Shushakova N, Haller H, Dumler I et al. Urokinase receptor mediates mobilization, migration, and differentiation of mesenchymal stem cells. Cardiovasc Res 2011; 90: 113-121.

40. Yoon BS, Moon JH, Jun EK, Kim J, Maeng I, Kim JS et al. Secretory profiles and wound healing effects of human amniotic fluid-derived mesenchymal stem cells. Stem Cells Dev 2010; 19: 887-902.

41. Kawai K, Xue F, Takahara T, Kudo H, Yata Y, Zhang W et al. Matrix metalloproteinase-9 contributes to the mobilization of bone marrow cells in the injured liver. Cell Transplant 2012; 21: 453-464.

42. Hamidouche Z, Fromigue O, Ringe J, Haupl T, Marie PJ. Crosstalks between integrin alpha 5 and IGF2/IGFBP2 signalling trigger human bone marrow-derived mesenchymal stromal osteogenic differentiation. BMC Cell Biol 2011; 11: 44.

43. Miyoshi S, Flexman JA, Cross DJ, Maravilla KR, Kim Y, Anzai Y et al. Transfection of neuroprogenitor cells with iron nanoparticles for magnetic resonance imaging tracking: cell viability, differentiation, and intracellular localization. Mol Imaging Biol 2005; 7: 286-295.

44. Egea V, Zahler S, Rieth N, Neth P, Popp T, Kehe K et al. Tissue inhibitor of metalloproteinase-1 (TIMP-1) regulates mesenchymal stem cells through let-7f microRNA and Wnt/beta-catenin signaling. Proc Natl Acad Sci USA 2012; 109: E309-E316.

45. Hamidouche Z, Fromigue O, Ringe J, Haupl T, Marie PJ. Crosstalks between integrin alpha 5 and IGF2/IGFBP2 signalling trigger human bone marrow-derived mesenchymal stromal osteogenic differentiation. BMC Cell Biol 2010; 11: 44.

46. Zhou L, Wang L, Lu L, Jiang P, Sun H, Wang H. Inhibition of miR-29 by TGF-beta-Smad3 signaling through dual mechanisms promotes transdifferentiation of mouse myoblasts into myofibroblasts. PLoS One 2012; 7: e33766.

47. Briassouli P, Rifkin D, Clancy RM, Buyon JP. Binding of anti-SSA antibodies to apoptotic fetal cardiocytes stimulates urokinase plasminogen activator (UPA)/uPA receptor-dependent activation of TGF-beta and potentiates fibrosis. J Immunol 2011; 187: 5392-5401.

48. Sakao S, Taraseviciene-Stewart L, Cool CD, Tada Y, Kasahara Y, Kurosu K et al. VEGF-R blockade causes endothelial cell apoptosis, expansion of surviving CD34 + precursor cells and transdifferentiation to smooth muscle-like and neuronal-like cells. FASEB J 2007; 21: 3640-3652.

49. Su L, Zhao J, Zhao BX, Zhang SL, Miao JY. Safrole oxide induces human umbilical vein endothelial cell transdifferentiation to 5-hydroxytryptaminergic neuron-like cells through tropomyosin receptor kinase A/cyclooxygenase 2/nuclear factor-kappa B/interleukin 8 signaling. Int J Biochem Cell Biol 2011; 43: 1512-1522.

50. Samarakoon R, Higgins CE, Higgins SP, Higgins PJ. TGF-beta1-induced expression of the poor prognosis serpine1/pai-1 gene requires egfr signaling: a new target for anti-egfr therapy. J Oncol 2009; 2009: 342391.

51. Lian N, Lin T, Liu W, Wang W, Li L, Sun S et al. Transforming growth factor beta suppresses osteoblast differentiation via the vimentin activating transcription factor 4 (ATF4) axis. J Biol Chem 2012; 287: 35975-35984.

52. Bakin AV, Rinehart C, Tomlinson AK, Arteaga CL. p38 mitogen-activated protein kinase is required for TGFbeta-mediated fibroblastic transdifferentiation and cell migration. J Cell Sci 2002; 115: 3193-3206.

53. Lee YJ, Lee HJ, Choi SH, Jin YB, An HJ, Kang JH et al. Soluble HSPB1 regulates VEGFmediated angiogenesis through their direct interaction. Angiogenesis 2012; 15: 229-242.

54. Hammond DE, Hyde R, Kratchmarova I, Beynon RJ, Blagoev B, Clague MJ. Quantitative analysis of HGF and EGF-dependent phosphotyrosine signaling networks. J Proteome Res 2010; 9: 2734-2742

55. Kratchmarova I, Blagoev B, Haack-Sorensen M, Kassem M, Mann M. Mechanism of divergent growth factor effects in mesenchymal stem cell differentiation. Science 2005; 308: 1472-1477.

56. Vuoriluoto K, Haugen H, Kiviluoto S, Mpindi JP, Nevo J, Gjerdrum C et al. Vimentin regulates EMT induction by Slug and oncogenic $\mathrm{H}$-Ras and migration by governing $\mathrm{Axl}$ expression in breast cancer. Oncogene 2011; 30: 1436-1448.

57. Kadri T, Lataillade JJ, Doucet C, Marie A, Ernou I, Bourin P et al. Proteomic study of Galectin1 expression in human mesenchymal stem cells. Stem Cells Dev 2005; 14: 204-212.

58. Takahashi K, Yamanaka S. Induction of pluripotent stem cells from mouse embryonic and adult fibroblast cultures by defined factors. Cell 2006; 126: 663-676.

59. Roobrouck VD, Clavel C, Jacobs SA, Ulloa-Montoya F, Crippa S, Sohni A et al. Differentiation potential of human postnatal mesenchymal stem cells, mesoangioblasts, and multipotent adult progenitor cells reflected in their transcriptome and partially influenced by the culture conditions. Stem Cells 2011; 29: 871-882.

60. Conrad S, Renninger M, Hennenlotter J, Wiesner T, Just L, Bonin M et al. Generation of pluripotent stem cells from adult human testis. Nature 2008; 456: 344-349.

cc) (i) $(\Theta)$ Cell Death and Disease is an open-access journal published by Nature Publishing Group. This work is licensed under a Creative Commons Attribution-NonCommercialNoDerivs 3.0 Unported License. To view a copy of this license, visit http://creativecommons.org/licenses/by-nc-nd/3.0/ 